\title{
MANUFACTURE OF MIXED ETHYL ESTERS OF HYDNOCARPUS-GROUP OILS.
}

Reprinted from the Report of the Leonard Wood Memorial Conference on Leprosy held in Manila, Philippine Islands.

Preparation of esters by hot process.-Ten litres of hydnocarpus oil are boiled vigorously for forty-eight hours with 5 litres of 96 per cent. ethyl alcohol and 100 cubic centimetres of concentrated sulphuric acid under a reflux condenser. The principal advantage of vigorous boiling is undoubtedly that it keeps the two layers well mixed. The esters are then separated from the lower layer from which the excess alcohol can be recovered by distillation. The esters are washed three times with about 20 litres of water, separated from the washing water, and dried in a steam kettle or on a water bath. The yield from 10 litres of oil is about 11 litres of esters. Proportionally smaller amounts of materials may be used to prepare smaller lots of esters.

Distillation.-The washed and dried ethyl esters are then distilled at 20 to 25 millimetres pressure in a glass or iron still.

Washing with alkali.-Before neutralising the esters, the acidity is determined by titration. Five hundred grams of lye (94 per cent. sodium hydrate) are dissolved in 80 litres of hot water $\left(90^{\circ}\right.$ C. $)$ in a 160-litre steel drum. Forty-five litres of the distilled ethyl esters ( 2.5 to 3 per cent. acidity) are added and thoroughly mixed. If the acidity is higher than 3 per cent. a correspondingly increased amount of lye is added. After the mixture has stood for twenty-four hours the clear lower layer is drawn off through an outlet in the bottom of the tank. Hot water $\left(90^{\circ} \mathrm{C}\right.$.) is added to the 140-litre mark, thoroughly mixed, allowed to settle for twenty-four hours, and again drawn off. The washing with water is repeated four times, and each time twentyfour hours are allowed for settling. The yield is about 96 per cent., and the acidity (as oleic acid) is usually under 0.1 per cent. If smaller lots of esters are to be neutralised 
it may be found necessary to add crystals of sodium chloride to assist the separation. Heating on a boiling-water bath will also hasten the process.

Steaming and drying.-Volatile impurities, which are of ten irritating, may be eliminated by blowing out with steam. The esters are placed in ordinary 5 gallon oil tins to settle, poured off from the separated water, and filtered through paper. The product is a clear, very pale yellow, mobile liquid with a slight characteristic odour. Smaller quantities of esters may be refined by this method.

Ethyl esters manufactured by the above process are of constant composition and relatively non-irritating, regardless of the source or quality of the original oil.

Preparation of esters by cold process.-Four hundred twenty-five grams of cold-drawn hydnocarpus oil of good quality, 550 cubic centimetres of 96 per cent. ethyl alcohol, and 32 cubic centimetres of sulphuric acid (sp. gr. 1-845) are placed in a bottle with a tight-fitting glass stopper and left until the process of esterification is complete. The bottle should be shaken once or twice a day to mix the upper and lower layers. This, or placing the bottle in the sun or in some warm place, hastens the process. Neither shaking nor heat is, however, essential if time is not a consideration. At first the oil forms a lower and the alcohol and acid an upper layer. As esterification proceeds a point is reached at which the lower layer, now chiefly composed of esters, is of lower specific gravity than and rises above, the original upper layer, which now contains a large proportion of glycerol. To ensure the completion of esterification it is well to allow the process to continue for a further period equal to the time required for the rising of the lower layer. Thus, if the lower layer takes fourteen days to rise, the ingredients should be left in the bottle for another fourteen days. When the upper layer dissolves completely in alcohol the esterification is complete; esters, but not oil, being completely soluble in alcohol. The lower layer is then drawn off and the upper layer repeatedly washed with an equal volume of water until the water is free from acid as tested by litmus paper. A 0.1 per cent. solution of sodium hydrate in water is then added; this forms a thick emulsion. The vessel is slowly rotated and crystals of common salt are gradually added in small quantities in order to break the enulsion. The lower layer is removed; and the upper layer consisting of esters, after being washed once more with distilled water, is filtered through thick filter paper. The esters, though now clear, still contain a 
certain amount of fine emulsion which makes them dark in colour. This may be removed by drying on a water bath for two or three hours while stirring constantly with a glass rod. The esters are then filtered again and the process is complete.

The esters may be washed in the bottle in which they have been prepared by substituting for the glass stopper a perforated cork with two glass tubes; one, 2 inches in length, is inserted flush with the inner end of the cork and fitted with a piece of rubber tubing compressed with a spring clamp; the other reaches to the bottom of the bottle. By inverting the bottle the esters rise to the top and the lower layer may be drained off by opening the clamp; or the lower layer may be syphoned off through the long glass tube without inverting the bottle. A separating funnel is more convenient for separating and washing the esters.

Preparation of ethyl esters by hot process without distillation.-The esters may be much more rapidly prepared by placing the ingredients mentioned under the cold process in a flask on a water bath arranged to maintain a constant level of water. A reflux condenser is connected with the flask. The water bath is kept at a temperature sufficient to maintain brisk boiling inside the flask. This is continued without stopping for eighteen hours, when it will be found that esterification is complete. Washing off the esters is then carried out as described in the cold process. The weight of the esters recovered is almost equal to that of the oil used.

In order to produce non-irritating ethyl esters by the last two processes, the original oil must be non-irritating.

*Addition of iodine.-Addition of metallic iodine $(0.5$ per cent.) to the ethyl esters markedly reduces the irritating quality of the ethyl esters. Fifteen litres of the purified esters are heated in a 20 litre enamelled kettle to $140^{\circ} \mathrm{C}$. The esters must be thoroughly dried before iodine is added since, if water is present, it effects by catalysis the hydrolysis of several per cent. of the esters. If the filtered esters are clear, the heating to $140^{\circ} \mathrm{C}$. before adding the iodine will drive off all dissolved water. Seventy-five grams of chemically pure re-sublimed iodine are added with stirring. The temperature immediately rises to $150^{\circ} \mathrm{C}$. at which point it is maintained for exactly thirty minutes, the liquid being stirred occasionally. After cooling, the iodised esters are filtered into bottles (250 cubic centimetres capacity) and sterilised for one hour in an oven at $150^{\circ} \mathrm{C}$. The temperature of the contents of the bottles reaches in this time $110^{\circ} \mathrm{C}$.

*Instead of iodine pure double distilled creosote to the extent of $4 \%$ may be added. - Editor. 
The bottles are tightly corked, and sealed with paraffin or sealing wax and allowed to stand two weeks before use.

Note 1.-Smaller lots of esters can be iodised by this method provided that a shallow (pan) type of container is used.

Note 2.- It is not advisable to use esters after the lapse of two years, although samples that have stood longer than three years do not seem to have deteriorated sufficiently to become markedly irritating.

Note 3.--Iodised esters must be kept tightly corked and preferably in a dark, cool place. Repeated resterilisation should be avoided. Heat or sunlight in the presence of air produces a change in the iodised esters, which increases their irritating qualities to a marked exterit.

Refining of hydnocarpus-group oils.-Crude hydnocarpus oils are sometimes found to give pain upon injection. A bland, non-irritating oil may be produced by the following methods of refining.

Volatile impurities can be removed by passing steam through the oil for about an hour, either before or after washing with alkali. Sufficient steam is used to give an aqueous distillate of about one-fifth the volume of the oil. The amount of volatile impurities is very small, but the distillate has a strong odour.

The free fatty acids are removed by washing with a solution of caustic alkali, and the only difficulty is the separation of the pure oil from the resulting emulsion. The following method has proven satisfactory. One hundred and fifty litres of hot water (about $90^{\circ} \mathrm{C}$.) are run into a 400 litre steel drum provided with a faucet at the bottom; 0.5 kilogram of lye (94 per cent. sodium hydrate) is added, followed by 100 litres of hydnocarpus oil (not over 2.5 per cent. acidity), and thoroughly mixed. With oil of higher acidity a correspondingly increased amount of lye must be used.

After the emulsion has stood twenty-four hours the clear lower layer (about 125 litres) is drawn off. Hot water is run in, while stirring, up to the 350 litre mark.

After two days the slightly opalescent lower layer $(200$ litres) is drawn off. The washing with water is repeated four times and twenty-four hours are allowed for separation on each occasion.

Ninety-five litres of oil are obtained, with an acidity of not over 0.2 per cent. This oil is steamed as described above and filtered, while hot, through folded filter papers. The 
filtrate is dried by heating in an enamel-ware kettle, filtered again, and sterilised in bottles at $150^{\circ} \mathrm{C}$.

Some provision must be made for keeping the oil hot during the separation of the emulsion if smaller quantities are refined, otherwise the mixture cools too rapidly to allow separation to take place. 